\title{
ARTICLE
}

\section{A mechanistic model of tau amyloid aggregation based on direct observation of oligomers}

\author{
Sarah L. Shammas ${ }^{1, \star}$, Gonzalo A. Garcia ${ }^{1, \star}$, Satish Kumar ${ }^{2, \star}, \dagger$, Magnus Kjaergaard ${ }^{1, \dagger}$, Mathew H. Horrocks ${ }^{1}$,
} Nadia Shivji1, Eva Mandelkow2,3, Tuomas P.J. Knowles ${ }^{1}$, Eckhard Mandelkow ${ }^{2,3,4}$ \& David Klenerman ${ }^{1}$

Protein aggregation plays a key role in neurodegenerative disease, giving rise to small oligomers that may become cytotoxic to cells. The fundamental microscopic reactions taking place during aggregation, and their rate constants, have been difficult to determine due to lack of suitable methods to identify and follow the low concentration of oligomers over time. Here we use single-molecule fluorescence to study the aggregation of the repeat domain of tau (K18), and two mutant forms linked with familial frontotemporal dementia, the deletion mutant $\Delta \mathrm{K} 280$ and the point mutant P301L. Our kinetic analysis reveals that aggregation proceeds via monomeric assembly into small oligomers, and a subsequent slow structural conversion step before fibril formation. Using this approach, we have been able to quantitatively determine how these mutations alter the aggregation energy landscape.

\footnotetext{
${ }^{1}$ Department of Chemistry, University of Cambridge, Lensfield Road, Cambridge CB2 1EW, UK. ${ }^{2}$ DZNE, German Center for Neurodegenerative Diseases, Ludwig-Erhard-Allee 2, 53175 Bonn, Germany. ${ }^{3}$ CAESAR Research Center, Ludwig-Erhard-Allee 2, 53175 Bonn, Germany. ${ }^{4}$ Max-Planck-Institute for Metabolism Research, Hamburg Outstation, c/o DESY, Notkestrasse 85, 22607 Hamburg, Germany. * These authors contributed equally to this work. $\dagger$ Present addresses: Institute of Forensic Science, Gujarat Forensic Sciences University, Gandhinagar-382007, Gujarat, India (S.K.); iNANO, Aarhus University, DK-8000 Aarhus C, Denmark (M.K.). Correspondence and requests for materials should be addressed to S.L.S. (email: sls42@cam.ac.uk) or to D.K. (email: dk10012@cam.ac.uk).
} 
T he spontaneous aggregation of proteins to form larger structures is a widespread and fundamental process in both normal and aberrant biology. Formation of large insoluble fibrils, often via self-assembly of soluble protein into oligomeric structures, is key to the pathology of several well-known human conditions such as Parkinson's disease ${ }^{1}$, prion disorders and Alzheimer's disease ${ }^{2}$. Alzheimer's disease is characterized by the deposition of two distinct types of aggregates-extracellular plaques composed of $\mathrm{A} \beta$ peptides (cleavage products of the transmembrane protein APP) and intracellular neurofibrillary tangles composed of hyperphosphorylated tau protein. Tau is a microtubule-binding protein that can aggregate into filaments, which are amyloid in nature (based on cross- $\beta$ structure) and are the major constituents of neurofibrillary tangles in the neurons of Alzheimer-diseased brains ${ }^{3}$.

Tau proteins are broadly speaking divided into two domainsa carboxyl-terminal microtubule-binding domain and an aminoterminal projection domain ${ }^{4}$. The microtubule-binding domain is positively charged overall, assisting its interaction with the negatively charged surfaces of microtubules, and contains three or four similar, but not identical, repeat regions of 31 or 32 amino acids ${ }^{5}$ (Fig. 1). Tau is rich in polar amino acids, which renders it a highly soluble protein with little secondary structure ${ }^{6}$ even once bound to the microtubule ${ }^{7}$. At first glance, it is therefore a surprise that this protein assembles into amyloid structures. However, hexapeptide motifs, which are present in two of the four repeat regions of the microtubule-binding domain, have a high $\beta$-sheet-forming propensity ${ }^{8}$. These have been demonstrated to be fundamental to the aggregation process, and form the core of the filaments 8 .9. Aggregation of full-length tau in vitro is indeed generally slow because of the charged, highly soluble nature of the protein ${ }^{10}$. In vitro aggregation studies have therefore tended to focus on constructs formed from the aggregation-prone repeat domain of the microtubule-binding domain (K18 and K19), which aggregate faster without the presence of the flanking regions ${ }^{11}$. In addition, polyanion cofactors such as heparin and RNA have been found to accelerate aggregation, presumably by

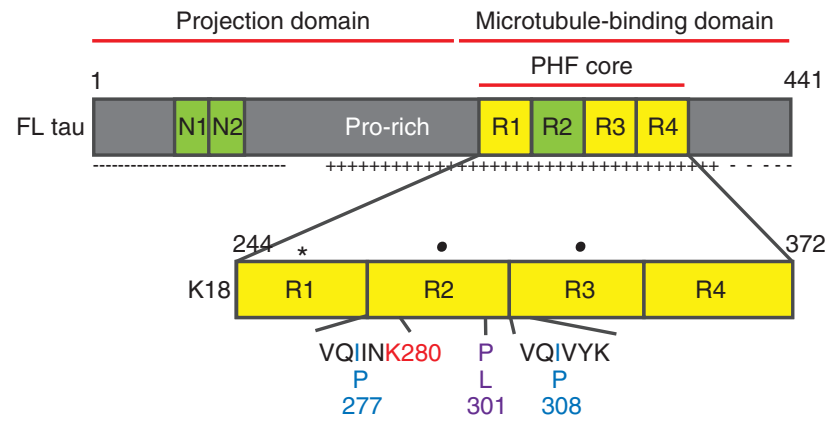

Figure 1 | Cartoon of the full-length tau sequence, showing the major regions and location of the $\mathbf{K 1 8}$ sequence. Tau is alternatively spliced in vivo, with two possible inserts near the $\mathrm{N}$ terminus ( $\mathrm{N} 1$ and $\mathrm{N} 2$ ) and one in the carboxyl-terminal half (R2). Repeats 1-4 (R1-R4), each 31 or 32 residues, represent the core of the filaments. The amino-terminal half of tau is termed 'projection domain' because it does not bind to the microtubule wall. The point deletion $\Delta K 280$ (indicated in red) located in one of the two hexapeptide motifs responsible for aggregation of tau into filaments ${ }^{43}$, and the substitution P301L (indicated in purple) that occur in FTD strongly enhance the $\beta$-sheet-forming propensity. The anti-aggregant PP mutant contains two substituted proline residues at positions 1277 and 1308 (indicated in blue). The $\mathrm{K} 18$ construct $^{11}$, consisting of the four repeat regions, has been adapted for this work through three mutations-C291A and C322A (black dots) and $1260 \mathrm{C}$ (black star) to allow specific fluorophore Alexa labelling at position 260 using maleimide chemistry. interacting with tau and compensating for the positive charges of the tau molecules, creating less unfavorable long-range electrostatic forces between tau molecules, and increasing the local tau concentration ${ }^{6,12-14}$. Heparin has traditionally been used to initiate aggregation within in vitro tau aggregation studies $^{14}$.

Until relatively recently, only average populations of fibrils were readily accessible in both in vivo and in vitro experiments, with transient oligomer populations proving much more difficult to detect ${ }^{15}$. To date, most theoretical modelling and understanding has thus focused on describing fibril formation, leading to some successful analytical treatments ${ }^{16,17}$. However, recent developments in single-molecule fluorescence techniques have led to significant advances in our ability to also detect the formation of lowly populated oligomeric species. New theoretical models are now desired that are capable of fully describing these better-characterized aggregation kinetics, and quantifying them. Although early single-molecule observations of synuclein aggregation were described with a simple conversion model ${ }^{18}$, an explicit kinetic treatment providing rate constants for all microscopic steps in the entire aggregation reaction has not been achieved to date. In this work, we have applied kinetic analysis to single-molecule fluorescence measurements to study the aggregation of tau protein and determine the aggregation pathway and the number and size of tau oligomers formed during this process. This approach allows the detailed analysis and quantification of many aspects of aggregating systems that may be difficult to directly measure experimentally.

\section{Results}

Aggregation of K18 tau into paired helical filaments. We have performed single-molecule fluorescence studies of AlexaFluor488 and AlexaFluor-647 labeled tau proteins (K18 construct) during their aggregation into filaments. This construct contains the four repeated sequences in the microtubule-binding domain that form the core of filaments and includes the two hexapeptide motifs in repeats R2 and R3, which nucleate aggregation ${ }^{3}$. We then compared its aggregation behaviour with that of a single point deletion mutant $(\Delta \mathrm{K} 280)$, one of the tau mutations found in frontotemporal dementia and parkinsonism linked to chromosome 17 (FTDP-17) ${ }^{19,20}$, and a single point mutation mutant $(\mathrm{P} 301 \mathrm{~L})$ that is the most common mutation associated with FTDP-17 (ref. 21). Fluorophore-labelled tau constructs based on the K18 tau construct were created (Fig. 1) ${ }^{10,11}$. Our results from bulk studies indicate that the presence of the Alexa label at residue 260 (outside the filament core) does not significantly affect the aggregation process; filaments formed were visibly similar by transmission electron microscopy (Supplementary Fig. 1) and aggregation rates were similar (Supplementary Fig. 2). We first measured the overall progression of the association reaction using ThT fluorescence and soluble protein concentrations. Under these conditions, filament formation is $\sim 90 \%$ complete within $3 \mathrm{~h}$ (Fig. $2 \mathrm{~b}$ ).

A single-molecule fluorescence resonance energy transfer (FRET) approach was then used to observe any oligomeric species $^{18}$. An equimolar mixture of A488-K18 and A647-K18 was incubated under filament-forming conditions. At various points throughout incubation, aliquots were removed and diluted to concentrations suitable for single-molecule fluorescence analysis. These solutions were then pumped under continuous flow through a microfluidic channel mounted on a microscope slide $^{22}$ (Fig. 2). A blue $(488 \mathrm{~nm})$ laser beam was focused to a diffraction-limited confocal spot within the centre of the microfluidic channel, leading to the excitation of A488-K18. Under these conditions, A647-K18 monomers were undetectable, 
a

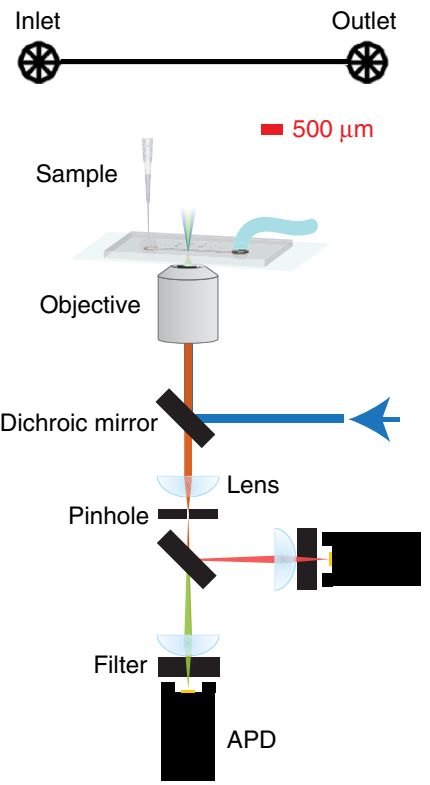

b
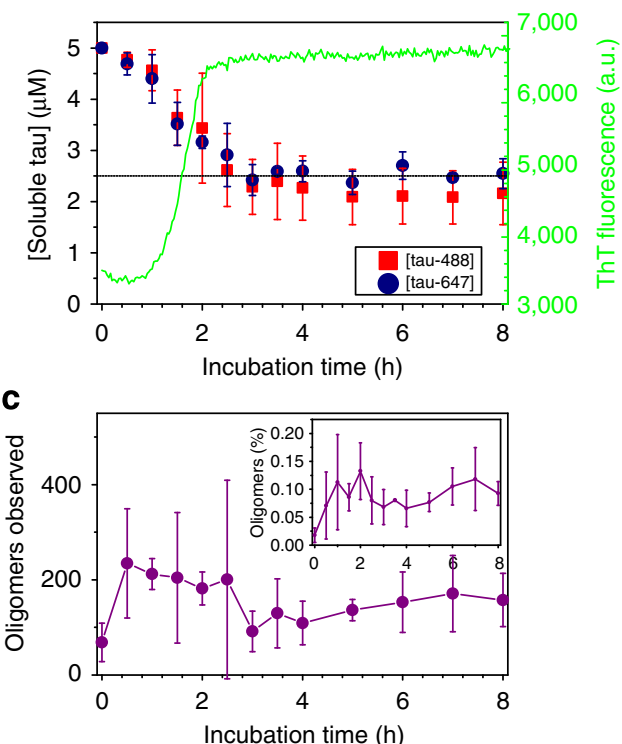

Figure 2 | Aggregation of K18 under standard fibril-forming conditions followed using single-molecule FRET. (a) Diagram of microfluidic channel (upper) and single-molecule FRET set-up (lower) used in this work. (b) Bulk aggregation kinetics for the filament formation of K18 (left-hand axis). Soluble tau concentrations of A488-K18 and A647-K18 during the experiment. The dotted line represents the remaining soluble concentration of unlabelled $\mathrm{K} 18$ after $24 \mathrm{~h}$ when it is incubated under the same conditions (right-hand axis, green line). Formation of filaments by A647-K18 monitored by ThT fluorescence. (c) Number of detected oligomers throughout the aggregation. The inset is the fraction of soluble tau in oligomers, according to burst rates. Aliquots of reaction mixture were diluted rapidly and subjected to single-molecule FRET analysis. In $\mathbf{b}, \mathbf{c}$, each point represents the average of - three to five individual measurements, and the error bars the s.d.

whereas A488-labelled monomers resulted in a single fluorescent burst in the blue channel. Oligomers containing both the labels were detected as bursts in the red channel, since any A647-K18 in close proximity to A488-K18 can be indirectly excited via FRET. We used this approach to quantify the proportion of the soluble (monomeric and oligomeric) material that was oligomeric. The vast majority of species observed were monomeric throughout the reaction. However, after only $0.5 \mathrm{~h}$, where $\sim 95 \%$ of tau remained soluble, there was an increase in the number of oligomers (Fig. 2c), representing $\sim 0.1 \%$ of the detected soluble tau species by number (Fig. 2c, inset). Most of the oligomers were only slightly brighter than the average monomer, and thus presumably relatively small. We estimated their apparent size by comparing the intensity of the oligomer fluorescence with that of a monomer, making the assumption that there was no significant quenching in the oligomeric species (see Methods) ${ }^{18}$. Pooling of all the data revealed that these small oligomers are most highly populated during the timeframe over which the filaments are formed and extended (Fig. 3, Supplementary Fig. 3). After $\sim 3 \mathrm{~h}$, the majority of filament formation had taken place, and the oligomer concentration appeared stable.

Observation of such a lowly populated species, which would certainly not be detected using traditional ensemble based methods, demonstrates the remarkable sensitivity of the technique for the early stages of the aggregation process. However, since the overall proportion of soluble tau in oligomers was so low, we took steps to ensure that we were detecting true oligomers in our experiments by examining an 'anti-aggregant' mutant form of tau as a negative control. The K18-PP mutant has two proline residues introduced by substitution (I277P and I308P) to reduce its $\beta$-sheet-forming propensity and is reported in the literature to prevent tau aggregation ${ }^{23}$. Immediately following initiation of the reaction, the concentration of oligomers detected was around just $0.1 \mathrm{nM}$, as compared with typical oligomer concentrations of a
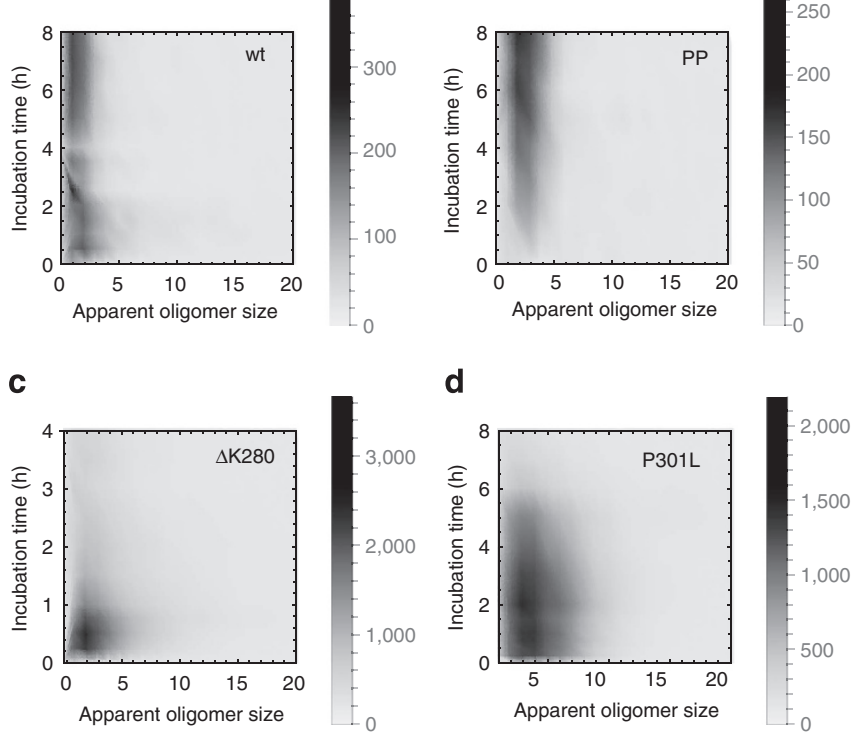

b

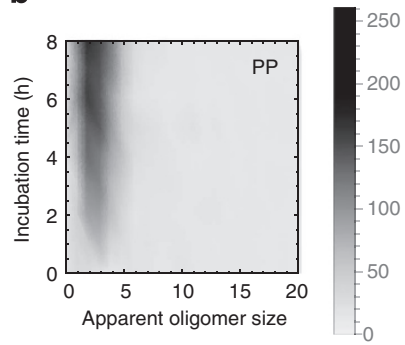

d

Figure 3 | Number density plots showing the evolution of the apparent oligomer size of A488/A647-K18 and mutants at different incubation times. Oligomers remain small throughout the reaction, with no observed especially stable sizes. Numbers reported are the total of each oligomer size recorded during the -three to five replicate experiments.

$5 \mathrm{nM}$ detected for wild-type K18, confirming that we are observing true oligomers in our experiments with wild-type tau (Fig. 4). Surprisingly, on incubation under our standard conditions, the number of oligomers detected increased steadily and significantly throughout the monitoring period of $8 \mathrm{~h}$ 


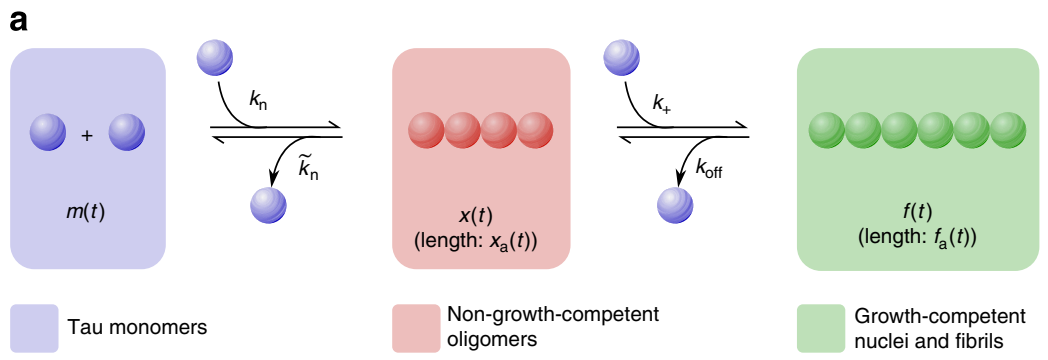

b
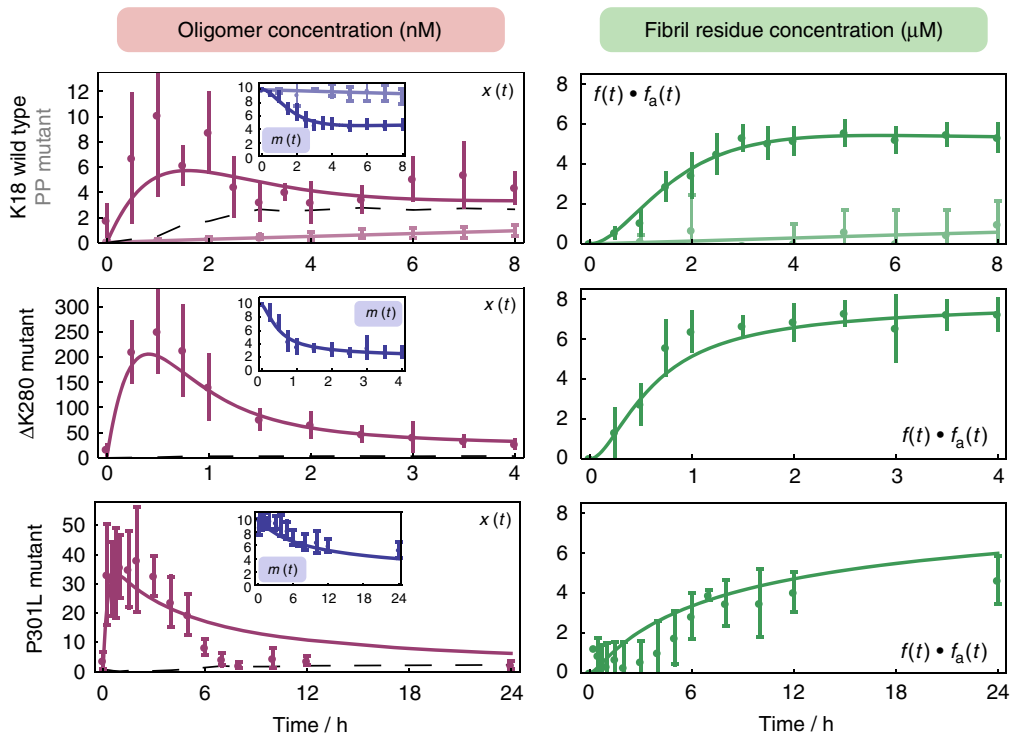

Figure 4 | Nucleation-conversion-polymerization description of tau aggregation. (a) Coarse-grained on-pathway conversion model obtained from theoretical considerations (Supplementary Material). Initial formation of non-growth-competent oligomeric species $x(t)$, of average length $x_{\mathrm{a}}(t)$, occurs via a reaction of order $n_{c}$ in monomer concentration $m(t)$. Formation of fibrils $f(t)$, of average length $f_{a}(t)$, then proceeds via addition of monomer units in a reaction of first order in $m(t)$. Rate constants $(k)$ for these processes and their corresponding reverse reactions are labelled. (b) Oligomeric and (deduced) fibrillar concentrations during the aggregation of K18, K18-PP, K18- $\Delta$ K280 and K18-P301L tau. Data represent the average of -three to five independent experiments, and error bars indicate the s.d. of these. Best fits for coarse-grained nucleation-conversion-polymerization description for K18, K18- $\Delta \mathrm{K} 280$ and K18-P301L are shown as solid lines. The dotted black curves indicate upper bounds for $x(t)$ predicted from the simpler inadequate nucleationpolymerization model (see Methods).

(Supplementary Fig. 4, $P<0.0001$ ), though at a much slower rate than that observed for wild-type tau, and without any apparent formation of filaments. Since we have not observed any filament formation, we do not know if these oligomers are of the same kind as those seen for K18-wt, or indeed if they are on- or offpathway; however, we note that they share a common size distribution with K18-wt (Fig. 3).

Disaggregation of K18 tau paired helical filaments. We also investigated the dissociation process of the labelled filaments formed under our standard aggregation conditions to demonstrate that the aggregation reaction was reversible. Filaments of A488/A647- K18 were collected by centrifugation, and fresh buffer added on top to initiate dissociation. The concentration of soluble tau increased with incubation time as the equilibrium between fibrillar and soluble tau was re-established. Immediately following dilution of the sample, a relatively large proportion $(0.6-3 \%)$ of the soluble tau was oligomeric (as compared with 0.1 $\%$ observed during aggregation); however, this proportion decreased rapidly during the first $24 \mathrm{~h}$ after dilution as the monomeric concentration increased, despite the overall oligomeric concentration increasing steadily over 1 month (Fig. 5). The oligomers observed in this experiment were nearly all small, consistent with the species being populated towards the end of the aggregation experiments.

Aggregation of two aggregation-prone K18 tau mutants. Having established a methodology, similar aggregation experiments were performed with the aggregation-prone deletion mutant of the $\mathrm{K} 18$ construct, $\mathrm{K} 18-\Delta \mathrm{K} 280$. The aggregation reaction took place faster under the same conditions as K18 (Fig. 4a), as has previously been reported ${ }^{20}$, being largely complete within 1-2 h, so we changed the separation of the data points accordingly. Again, the majority of soluble species were monomeric (Fig. 4). The total number of detected oligomers peaked at $0.5 \mathrm{~h}$, during the filament growth phase, and thereafter decreased, but remained higher than the number observed for K18-wt, even towards the end of the reaction. At peak, there were around 50 times more oligomers observed than for K18, where $\sim 4 \%$ of the species detected were oligomeric (Fig. 4b). Owing to their larger population, we are better able to characterize the oligomers for this version of $\mathrm{K} 18 \mathrm{tau}$, as demonstrated by the contour plots of the number distribution for K18- $\Delta \mathrm{K} 280$ (Supplementary Fig. 3b). Similar to K18-wt, the vast majority of the observed oligomers remain small throughout the reaction (Fig. 3). We did not observe any peaks in the intensity distribution that would have suggested 

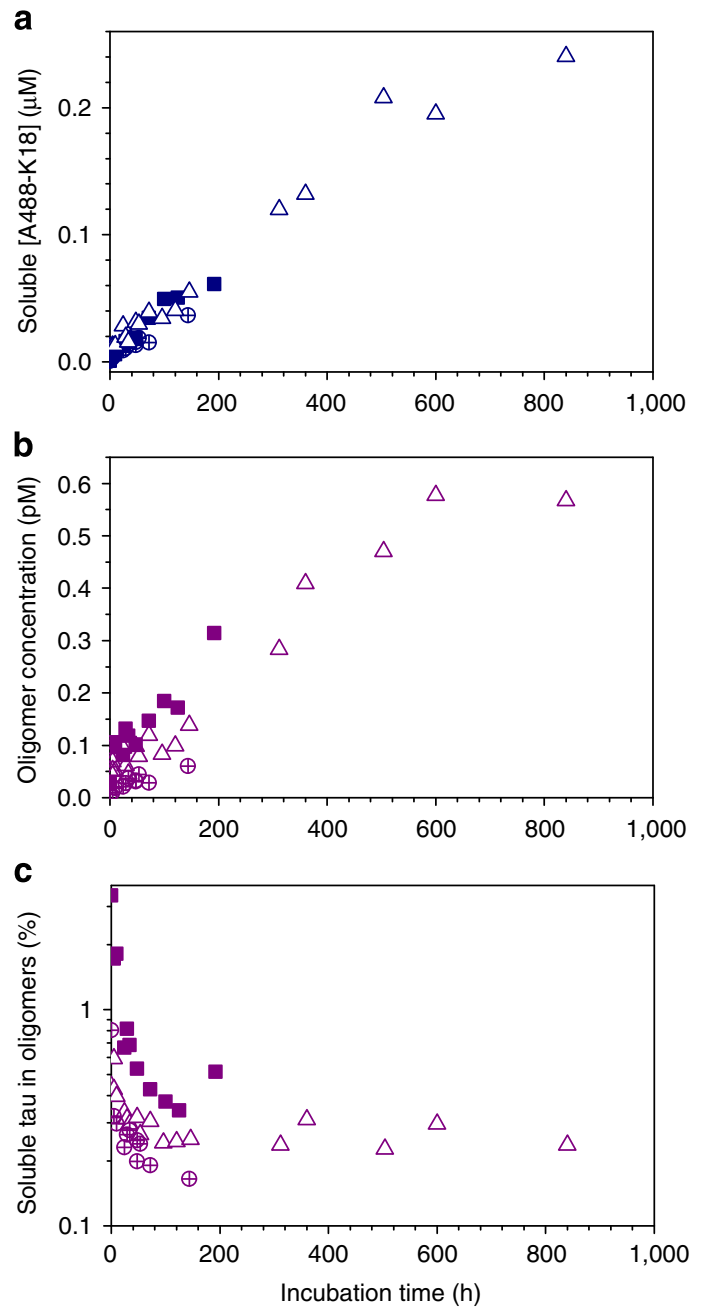

Figure 5 | Fibrils formed from A488-K18 and A647- K18 tau disaggregate slowly into oligomers and monomers. (a) Soluble concentrations of A488-K18 were estimated using the burst rate in the donor channel from smFRET measurements. (b) The proportion of events due to oligomeric K18 decreases with incubation time, although the estimated total number of oligomers within the solution increased. (c) Three independent experiments were performed (open, filled and crossed symbols), the initial filament mass was similar but not identical in each.

especially stable oligomer sizes; however, a small concentration of brighter species appear as the reaction proceeds and fibrils are created (Supplementary Fig. 3b). This could represent an increase in the average size of the oligomers or fibrils passing through the edges of the confocal spot, but represents a minority of the detected events.

Further aggregation experiments were then conducted with another mutated version of K18 associated with FTDP-17, K18-P301L. Oligomer concentrations reached a rapid peak $\sim 0.5 \mathrm{~h}$ after initiation of around-three to four times that observed for K18-wt, remaining high for some time before decreasing slowly as fibrils were formed (Fig. 4b). The oligomer size distribution is very consistent over the first few hours when few fibrils are present in the reaction mixture and oligomer concentrations are high, and strongly resembles those from both K18-wt and K18- $\Delta \mathrm{K} 280$ (Fig. 3).

The larger concentrations of oligomers formed by the $\mathrm{K} 18-\Delta \mathrm{K} 280$ mutant also allowed us to assess their stability on dilution. Oligomer-'rich' samples were prepared by incubating $\mathrm{K} 18-\Delta \mathrm{K} 280$ for $30 \mathrm{~min}$ under our standard conditions, and changes in the oligomer concentration following dilution were then monitored through the oligomer burst rate. The results of this experiment showed that some oligomers were dissociating throughout our typical measurement times, but that our estimated concentrations were underestimated by at most a factor of two (Supplementary Fig. 5).

Since earlier publications have reported on the toxic nature of small $\beta$-sheet-rich tau oligomers to the neuronal cell line SH-SY5Y ${ }^{24-26}$, we tested our samples of oligomer-containing $\mathrm{K} 18-\Delta \mathrm{K} 280$ for such toxicity using a similar approach. Application of oligomers to the cell samples resulted in no detectable toxicity (Supplementary Fig. 6) even though we found the oligomers to be stable in cell culture medium throughout the incubation times used (Supplementary Fig. 7), possibly due to the tau in our experiments not being full length.

Oligomeric species are observed for all K18 tau types, and their formation broadly precedes fibril formation in aggregation reactions, with oligomer populations sequentially rising and falling before bulk depletion of monomer. Therefore, a key mechanistic question asks how the different timescales and amplitudes of the processes are related. A simple nucleationpolymerization model ${ }^{16,17,27,28}$, treating oligomers as small fibrils, cannot describe the observed oligomer population amplitudes while permitting the observed formation of long fibrils (Supplementary Fig. 8 and Online Methods). However, if oligomeric species are considered to be structurally distinct from small fibrils, the necessary introduction of an on-pathway conversion reaction successfully describes the reaction kinetics, for both the K18 construct and the $\Delta \mathrm{K} 280$ and P301L mutants (Fig. 4).

Qualitative comparison of observations for K18 and its mutant versions highlights major differences between their kinetics. Oligomers are more highly populated for both (diseaseassociated) mutants, K18- $\Delta \mathrm{K} 280$ and P301L. The overall timescales for the reactions are also different, for both the emergence of oligomers and filament accumulation. In the case of $\mathrm{K} 18-\Delta \mathrm{K} 280$, the reaction reaches completion after $\sim 2 \mathrm{~h}$ compared with $4 \mathrm{~h}$ for K18, whereas the 'anti-aggregant' PP mutant, displays no noticeable accumulation of fibrils within $8 \mathrm{~h}$. It is also of note that although oligomers may have been observed for the 'anti-aggregant' PP mutant, the oligomer concentrations have still not reached their peak within $8 \mathrm{~h}$, suggesting that the nucleation rate constant is much lower for this mutant. In contrast, peak oligomer concentrations are reached rapidly for $\mathrm{P} 301 \mathrm{~L}$ and $\mathrm{K} 18-\Delta \mathrm{K} 280$, suggesting that nucleation is significantly more favourable for these mutant tau species in comparison with wild type. We sought to describe these results in a more quantifiable fashion. Detailed quantification of these differences is made possible by numerically fitting our simple conversion model where monomers associate to form oligomers (of the apparent size observed in our experiments) in a heparin-dependent manner, and are subsequently converted into fibrillar species (Fig. 4a). Fitting to each of the data sets allowed the extraction of all relevant rate constants (Fig. 4 b, Supplementary Table 1), and subsequently the changes in the activation free energy introduced on mutation for each reaction step, as described in Methods and summarized in Table 1 . Since we had observed that there was some dissociation of oligomers during our measurements, we established that the estimated activation free energy changes were insensitive to the exact oligomer concentrations, that is, values did not change within error when all oligomer concentrations were doubled (see Methods). Although several mutation effects are apparent, the most striking one is the large increase in the initial nucleation rate on $\Delta \mathrm{K} 280$ mutation. This effect primarily explains the higher number of oligomers observed for $\mathrm{K} 18-\Delta \mathrm{K} 280$ aggregation, and is likely to reflect reduced 


\begin{tabular}{|c|c|c|c|c|}
\hline Protein & $k_{n}$ & $\check{\boldsymbol{k}}_{n}$ & $\boldsymbol{k}_{+}$ & $k_{\text {off }}$ \\
\hline$\Delta \mathrm{K} 280$ & $-4.9 \pm 0.7$ & $-1.5 \pm 0.7$ & $+2.5 \pm 0.6$ & $+1.7 \pm 1.0$ \\
\hline P301L & $-2.7 \pm 0.7$ & $-1.8 \pm 0.7$ & $+3.1 \pm 0.8$ & $+4.6 \pm 1.1$ \\
\hline PP & $4.1 \pm 0.7$ & ND & ND & ND \\
\hline
\end{tabular}

ND, not determined; PP, I277P I308P mutant.

The change in activation energies (from K18-wt) for the forward and backward processes of nucleation to form oligomers from monomer $\left(k_{n}\right.$ and $\left.\check{k}_{n}\right)$ and the conversion of oligomers into fibrils $\left(k_{+}\right.$and $\left.k_{\text {off }}\right)$ are reported in units of RT for each mutant.

electrostatic repulsion between monomers due to its reduced overall charge in comparison with $\mathrm{K} 18$ and/or its greater propensity to form $\beta$-structure ${ }^{23}$. The 50 -fold increase in oligomer population only results in an approximately twofold reduction in the timescale of fibril formation, suggesting an additional effect of the mutation of the transition from oligomers to fibrils. Accordingly, the activation free energy for fibril formation from oligomers also increases on mutation.

Similar results were also obtained for the P301L mutant, with nucleation (and denucleation) rates being increased, but fibril elongation (and shortening) rates being decreased compared with wild type (Table 1). Although appropriate for early times, the fit for this mutant does not describe the later stages of the aggregation process so successfully, indicating the possible presence of additional processes not included in our simplified scheme. However, it remains useful for comparative purposes, so has been used to make approximate quantitative predictions alongside the other proteins studied. Although we are not able to extract reliable estimates for all rate constants for K18-PP, we can make a robust estimate of the change in the nucleation rate on mutation and this is significantly reduced (Table 1).

\section{Discussion}

Interestingly, the two mutants with increased $\beta$-sheet-forming propensity display increased nucleation rates, but reduced fibril elongation (and shortening) rates, while the mutant with decreased $\beta$-sheet-forming propensity (K18-PP) has a reduced nucleation rate. Although we observed no apparent toxicity (by MTT (3-(4,5-dimethylthiazol-2-yl)-2,5-diphenyltetrazolium bromide) or lactate dehydrogenase assays of K18 oligomers on incubation with neuronal cells in our model system (Supplementary Fig. 6) it is possible that these standard assays are too coarse to detect an effect. A recent study has shown tau oligomers to have more subtle effect by decreasing dendritic spine density of differentiated neurons ${ }^{29}$. We note that the nucleation rates we observed appear to correlate with aggregation of tau within the brain in inducible transgenic mice systems ${ }^{30,31}$.

There are several studies based on ensemble methodologies, such as light scattering or ThT fluorescence, of the effect of mutation in tau on aggregation kinetics. These studies are fundamentally quite different to that presented here because these methods being (broadly) sensitive to fibril concentration best report on the later stages of aggregation, rather than the earlier stages addressed here. Nonetheless, we have found that many of our results recapitulate these earlier findings. For example, it has been suggested that the $\Delta \mathrm{K} 280$ mutation leads to an increased nucleation rate and an enhanced overall aggregation kinetics ${ }^{10}$. Similarly, consistent with the literature, we did not observe any decrease in the concentration of soluble K18-PP tau throughout our measurements. We were, however, able to observe some oligomers, whose number increased during our monitoring period, but still remained lower (1/5th-1/ 10th) than for the wild-type protein. In general, it is agreed that P301L aggregates faster than wild-type tau ${ }^{20,32}$ and it has previously been suggested for full-length tau that this is due to faster fibril elongation ${ }^{33,34}$. Under the conditions used here, we observed that despite

the rapid formation of oligomers, the reaction approached steadystate more slowly on P301L mutation due to a reduction in the fibril elongation rate constant. There are differing reports of the effect on nucleation, all based on full-length tau, with suggestions that the mutation enhances ${ }^{32,33}$, or retards ${ }^{34}$ the process. Our technique, highly sensitive to small oligomeric species, has demonstrated that the nucleation rate is in fact increased due to the mutation, at least in the context of the K18 repeat construct.

It is well known that 'seeding', the addition of preformed fibrillar or oligomeric structures, generally increases the rate of aggregation in a number of systems, and this has been observed in vitro for tau $26,35,36$. However, experimentally quantifying this effect is challenging due to the difficulty in determining the concentration of these initial seeds. These complications are particularly prominent for oligomeric seeding due to added difficulties in detecting oligomer populations in general. Theoretical modelling thus provides a powerful alternative approach, allowing order-of-magnitude estimates for system properties that are experimentally inaccessible, via careful extrapolation of observations. On the basis of the general conversion model and fit parameters described in Fig. 4, we can estimate the concentration of oligomeric seeds required to double the rate of fibril formation under the experimental conditions described. By following the procedure described in Supplementary Material, we arrive at estimates of $0.01 \mu \mathrm{M}$ for $\mathrm{K} 18,0.3 \mu \mathrm{M}$ for $\mathrm{K} 18-\Delta \mathrm{K} 280$ and $0.2 \mu \mathrm{M}$ for K18-P301L. The difference in values reflects the extent to which the initial nucleation step limits the overall aggregation reaction for each tau construct; for $\mathrm{K} 18$, it represents an important bottleneck in the flow of mass from free monomer to fibrils, thus a relatively small contribution to this step via seeding has a larger overall effect. Similarly, we are able to estimate the effects of seeding under conditions of compartmentalization, one of a myriad of complicating factors that occur in the cellular environment. For a typical neuronal cell volume of $6,000 \mu^{3}$ at a tau concentration of $2 \mu \mathrm{M}$, the reaction half-time for K18 can be halved from an estimated 4 to $2 \mathrm{~h}$ by extremely low numbers of oligomers; $\sim 30$ oligomers suffice, under the assumption that the nucleation reaction is of second order in monomer such that $n_{\mathrm{c}}=2$. This indicates that only a very small number of seeds would need to be transferred from cell to cell for spreading of oligomers via a prion-like mechanism ${ }^{37,38}$, in an idealized situation without inhibition by cellular chaperones. For K18- $\Delta$ K280 and P301L under the same conditions, $\sim 2,000$ oligomers are required to approximately halve the reaction half-time from 4 to $2 \mathrm{~h}$ and 300 to $150 \mathrm{~h}$ respectively. Spreading is thus potentially less efficient for $\mathrm{K} 18-\Delta \mathrm{K} 280$, although a 100-fold higher concentration of oligomers, and a fourfold higher mass of fibrils, could be present in the cell as compared with K18, increasing the probability of cellular damage and death. Although these calculations are based on a shortened construct of tau and therefore miss much of the complexity of the biological situation, these calculations may be instructive in understanding the dynamics of spreading. These results are particularly compelling in light of recent results demonstrating differential spreading of tau isoforms between neurons in vivo ${ }^{21}$. Following injection of lentiviral particles encoding tau, pathology development for wildtype tau was spread throughout neurons connected to the injection site, whereas pathology associated with P301L tau was restricted to cells close to the injection site despite the higher level of neuronal death observed in the latter case ${ }^{21}$. 
In summary, we have combined single-molecule fluorescence and kinetic analysis to determine the aggregation pathway for the tetra-repeat K18 tau construct and two disease-related mutants, allowing us to determine how the mutation alters the aggregation pathway. Our quantitative kinetic model with microscopic rate constants allows us to show that seeding may be highly effective in the cellular environment, providing support for the prion-like spreading model. This could serve as a general approach to determine the critical molecular events that lead to the formation of potentially toxic oligomers, and additionally enables the number of oligomers and aggregation rate to be estimated under conditions that are not accessible experimentally.

\section{Methods}

Chemicals. Thioflavin T, ammonium acetate and bovine serum albumin (BSA) were all purchased from Sigma. AlexaFluor-488 C5 and 647 C2 maleimide and Vybrant MTT Cell Proliferation Assay Kit are produced by Molecular Probes. Heparin 3,000 was obtained from MP Biomedicals.

K18 and mutant K18 constructs. To ensure specific labelling, the two natural cysteine residues at positions 291 and 322 (in R2 and R3) were mutated to alanine, and a cysteine was introduced at position 260. This enabled fluorophore labelling of the protein without introducing bulky residues in the repeat domain responsible for aggregation. The construct (K18-C291A/C322A/I260C) was expressed in E. coli as described previously ${ }^{6}$, and labelled with either AlexaFluor- 488 or AlexaFluor647 using maleimide chemistry. Mass spectroscopy, SDS-polyacrylamide gel electrophoresis (SDS-PAGE) and analytical size exclusion all showed the protein labelling to have been successful, and the material to be apparently free of preformed aggregates (Supplementary Fig. 1). The aggregation rate of A647-K18 and A647-K18- $\Delta \mathrm{K} 280$ were similar to that of unlabelled K18 and K18- $\Delta \mathrm{K} 280$ in the presence of dithiothreitol (Supplementary Fig. 1). Described mutations were achieved through site-directed mutagenesis.

Aggregation of tau constructs. We employed the same standard aggregation conditions in all our assays to ensure comparability. Solutions of $10 \mu \mathrm{M} \mathrm{K18}$ construct in $50 \mathrm{mM} \mathrm{pH} 7.0$ ammonium acetate were incubated undisturbed at $37^{\circ} \mathrm{C}$, in the presence of a 1:4 molar ratio of heparin (MW 3000) as an initiator.

Thioflavin T measurements of tau aggregation. K18 construct was incubated under standard aggregation conditions in the presence of $20 \mu \mathrm{M}$ Thioflavin $\mathrm{T}$ and $5 \mathrm{mM}$ dithiothreitol in untreated half-area 96-well plates (Corning). The fluorescence intensity throughout incubation was monitored using a FLUOstar fluorescence platereader (BMG Labtech, Offenburg, Germany).

Soluble tau concentrations. In aggregation studies, soluble protein concentrations were monitored by periodic removal of aliquots, and 400 -fold dilution, followed by SDS-PAGE analysis. Fluorescence imaging of gels (using a Typhoon Trio, GE Healthcare) was used to quantify band intensity. Concentrations were calculated by comparison with the band intensity of the starting mixture, which was known to be $5 \mu \mathrm{M} \mathrm{A} 488-\mathrm{K} 18$ and $5 \mu \mathrm{M}$ A647-K18. The final concentration of soluble tau (roughly $5 \mu \mathrm{M}$ ) also matched that found for the unlabeled construct from SDS-PAGE analysis and Coomassie staining (Fig. 2b, dotted line). In aggregation studies of K18- $\Delta \mathrm{K} 280$, K18-P301L and K18-PP and disaggregation of $\mathrm{K} 18$, the burst rate in the donor channel (using an event threshold of 10) from single-molecule measurements was used as a probe of the concentration of soluble A488-K18. This approach was validated by comparison with results from quantitative SDS-PAGE analysis.

Single-molecule FRET measurements of K18 aggregation. Aliquots of aggregating mixtures of equimolar A488-K18 and A647-K18 were removed periodically without disturbing the pellet and diluted 50,000 -fold into $50 \mathrm{mM} \mathrm{pH} 7.0$ ammonium acetate buffer containing $0.1 \mathrm{mg} \mathrm{ml}^{-1}$ BSA and $2.5 \mu \mathrm{M}$ heparin (average MW 3,000). After dilution, the solutions were loaded into a gel-loading tip attached to the inlet of a simple one-channelled PDMS microfluidic device measuring $25 \mu \mathrm{m}$ in height and $100 \mu \mathrm{m}$ in width, as described previously ${ }^{22}$. Sample was passed through the channel by withdrawing solution from the device at the outlet channel at a flow rate of $0.5 \mathrm{~cm} \mathrm{~s}^{-1}$ (achieved by attaching the outlet to a syringe pump (PHD2000, Harvard Apparatus)). The device was mounted onto a home-built single-molecule confocal instrument, described in detail previously ${ }^{39}$. In brief, 488 -nm laser light at an intensity of $2 \mathrm{~mW}$ was directed through the back port of an inverted microscope (Nikon Eclipse TI) where it was reflected by a dichroic mirror (FF500/646-Di01) through an oil immersion objective (Nikon CFI Plan Apochromat VC 60X Oil N2 NA 1.4, W.D $0.13 \mathrm{~mm}$ ), which focuses it to a diffraction limited confocal spot within the microfluidic channel. The emitted fluorescence is collected by the same objective and passes through the dichroic mirror, before being focused by a tube lens within the microscope body through a $50 \mu \mathrm{m}$ pinhole (Thorlabs). A dichroic mirror (585DRLP Horiba) then separates the fluorescence from the two different fluorophores; the longer wavelength passes through the dichroic and is focused by a lens (Plano apo convex, focal length $=50 \mathrm{~mm}$, Thorlabs) through a band-pass and long-pass filter 565ALP/ 695AF55 (both Horiba) onto the Avalanche Photodiode (APD) detector. The shorter wavelength is reflected by the dichroic and is focused through a long-pass and band-pass filter 535AF45 (Horiba)/540LP (Omega optics) onto the second APD. Outputs from the two APDs are connected to a custom-programmed fieldprogrammable gate array, FPGA (Colexica), which counts the signals and combines them into time bins of $100 \mu \mathrm{s}$, which matches the expected residence time of the molecules in the confocal volume.

Analysis of single-molecule FRET aggregation measurements. The time-binned data were analysed using custom-written software in Igor Pro (Wavemetrics), which corrects for both autofluorescence and cross-talk.

The total number of events (monomeric and oligomeric) due to donor (A488)labelled tau was determined by counting the number of bursts that exceeded 10 counts per bin in the donor channel and used to determine the concentration of soluble donor-labelled tau (by comparison with a solution of known tau concentration, that is, immediately after initiation of the reaction). This approach was validated by cross-checking against SDS-PAGE gels of pelleted samples (see soluble tau concentrations, Methods).

Oligomeric events were then identified as bursts that exceeded 10 counts per bin in the acceptor channel since monomeric tau labelled with either donor or acceptor (A647) would not appear in this channel. We performed experiments to assess the stability of the oligomers during our measurement procedure. 'Oligomer-rich' samples were generated by incubating $\mathrm{K} 18-\Delta \mathrm{K} 280$ for $30 \mathrm{~min}$ using our standard conditions and then diluted for $(20 \mathrm{~min})$ single-molecule fluorescence measurements. Under these conditions, the oligomer concentration is high enough to examine the oligomer burst rate throughout the measurement. Oligomer concentrations were found to gradually decrease throughout the measurement time within buffers containing BSA (used in our dilution buffer to prevent adsorption of labelled tau to surfaces); however, this affects the overall estimated concentration by less than a factor of two (Supplementary Fig. 5) and does not affect our estimates of the changes in activation energies. It is possible that unstable oligomers, which dissociate within the dead-time of our experiment (a few minutes), are also present in the aggregation reaction mixture; however, such unstable species seem unlikely to impart toxicity and are not required to create a mechanistic model that describes the data. Oligomer concentrations (by number, not mass) were estimated by multiplying the soluble concentration of tau (which is predominantly monomeric) by the proportion of detected events attributed to oligomers. A minor fraction of very intense events spanning multiple time bins was observed in a minority of K18 samples at late time points where sedimented fibrils had been disturbed and was ascribed to fibrillar fragments. To focus on soluble oligomers, these events were excluded from the analysis. Sedimentation was observed reproducibly for K18-wt samples a few hours after initiation of aggregation, but not for any of the K18 mutants. To check that oligomers were not being sequestered within an insoluble fraction and disturbing our analysis, we performed a control experiment where K18 was aggregated as previously but under conditions of continuous shaking to avoid sedimentation. Both the equilibrium monomer concentration and the oligomer concentrations were largely unaffected indicating that the oligomers were not being removed from the solution (Supplementary Fig. 9).

For each of the oligomeric events, the apparent size was calculated by combining the intensity remaining in the donor channel with that transferred to the acceptor channel (after application of a correction factor, $\gamma$, to account for the different quantum yields and detection efficiencies in the two channels) and comparing this with the average intensity of monomeric events observed in the donor channel. We have used this approach previously ${ }^{18}$, the apparent size being calculated using equation (1)

$$
\text { Apparent oligomer size }=2\left(\frac{I_{\mathrm{D}}^{\prime}+I_{\mathrm{A}}^{\prime} / \gamma}{<I_{\mathrm{D}}>}\right)
$$

where $I_{\mathrm{D}}^{\prime}$ and $I_{\mathrm{A}}^{\prime}$ correspond, respectively, to the intensity in the donor and acceptor channels for the oligomeric event, $\left\langle I_{D}\right\rangle$ represents the average intensity of donor labeled monomers in the donor channel, and the factor of 2 accounts for the fact that on average each oligomer is composed of only $50 \%$ donor-labelled tau. Although this last assumption is true on average, it is not true for all oligomers, and this acts to broaden estimated size distributions, particularly for small oligomers. We have previously seen good agreement between size distributions estimated using this approach and for the same oligomers immobilized on surfaces ${ }^{40}$ Following these calculations, events were binned by apparent oligomer size (bin width $=1$ ) and FRET efficiency (bin width $=0.05$ ), where the FRET efficiency is calculated according to equation (2):

$$
\text { FRET efficiency }=\frac{I_{\mathrm{A}}^{\prime} / \gamma}{I_{\mathrm{D}}^{\prime}+I_{\mathrm{A}}^{\prime} / \gamma}
$$


Single-molecule FRET measurements of filament dissociation. Labelled K18 filaments were prepared using the standard aggregation conditions, and then purified by four cycles of centrifugation where the pellet was washed by replacement of the supernatant with fresh $50 \mathrm{mM} \mathrm{pH} 7.0$ ammonium acetate buffer. Following the final wash, $40-65 \mu \mathrm{l}$ of fresh buffer was carefully pipetted above the pellet without disrupting it. Aliquots of solution (away from any pellet) were removed periodically, rapidly diluted into $50 \mathrm{mM} \mathrm{pH} 7.0$ ammonium acetate and placed on BSA-treated microscope slides (not assessed under flow conditions). These experiments were performed using a different instrument to the aggregation assays. The set-up, experimental and data analysis protocols have been described in detail previously 18,39 , and were subject to only the following minor modifications. The 488-nm laser was used at a power of $80-90 \mu \mathrm{W}$. We identified bursts above a threshold of 7 photons $\mathrm{ms}^{-1}$ bin in the acceptor channel as being due to oligomers. In support of this approach, buffer-only controls analysed in this manner typically resulted in only $\sim 1 \%$ of the number of events as those detected in dissociation reactions.

Cell culture thiazolyl blue tetrazolium bromide (MTT) assay. SH-SY5Y cells (from the European Collection of Cell Cultures) were cultured at $30 \times 10^{3}$ cells per well in a 96-well plate in DMEM supplemented with $10 \%(\mathrm{v} / \mathrm{v})$ fetal calf serum, $1 \%$ (v/v) penicillin/streptomycin in a $5 \% \mathrm{CO}_{2}$ environment at $37^{\circ} \mathrm{C}$ for $24 \mathrm{~h}$. After $24 \mathrm{~h}$, the medium was replaced with $50 \mu \mathrm{l}$ of fresh culture medium. Samples of $\mathrm{K} 18-\Delta \mathrm{K} 280$ were aggregated using the standard conditions to result in maximal concentration of oligomers $(0.5 \mathrm{~h}$ for K18- $\Delta \mathrm{K} 280)$. Dilutions of K18- $\Delta \mathrm{K} 280$ were made up in DMEM and then $50 \mu \mathrm{l}$ of sample was added to each well. A positive control of $5 \mu \mathrm{M}$ Staurosporin and negative controls of HBSS and medium alone were also added to the cells. The cells were left to incubate for $4 \mathrm{~h}$ in a $5 \% \mathrm{CO}_{2}$ environment at $37^{\circ} \mathrm{C}$. The media were then removed and $100 \mu \mathrm{l}$ of fresh medium was added to each well. Ten $\mu \mathrm{l}$ of $12 \mathrm{mM}$ MTT stock solution was then to each well and incubated at $37^{\circ} \mathrm{C}$ for $2 \mathrm{~h}$. All but $25 \mu \mathrm{l}$ of medium was then removed from the cells and $50 \mu \mathrm{l}$ of dimethylsulphoxide was added to each well and mixed thoroughly with a pipette. The cells were then incubated for $10 \mathrm{~min}$ at $37^{\circ} \mathrm{C}$. The plates were shaken for $1 \mathrm{~min}$ and absorbance at $540 \mathrm{~nm}$ for each sample determined with a FLUOstar Optima plate reader (BMG Labtech). Absorbance values of SH-SY5Y cells in different experimental conditions were normalized against cells treated with DMEM media.

Failure of classical nucleated polymerization theory. The classical theory of nucleated polymerization ${ }^{27,28}$ provides a simple yet extraordinarily powerful description of aggregating filamentous systems. With only minor modifications, this framework has led to successful descriptions for the kinetics of a wide range of proteins ${ }^{16,27,28,41,42}$. A notable simplification that renders these models so useful is the identification of only two species in the reaction pathway: monomers and fibrils. Any oligomeric species are treated simply as small fibrils, and off-pathway reactions are neglected.

The master equation describing the kinetics of a nucleation-polymerization reaction is illustrated in Fig. 5, and reads

$$
\begin{aligned}
\dot{f}(j, t)= & \delta_{j, n_{\mathrm{c}}} k_{\mathrm{n}} m(t)^{n_{c}}+2 k_{+} m(t) f(j-1, t)-2 m(t) k_{+} f(j, t) \\
& -2 k_{\text {off }} f(j, t)+2 k_{\text {off }} f(j+1, t),
\end{aligned}
$$

where $\delta_{x, y}$ denotes the Kronecker symbol. The critical nucleus size is denoted $n_{\mathcal{c}}$, with a corresponding nucleation rate constant $k_{\mathrm{n}} ; n_{\mathrm{c}}$ represents the number of monomer residues that make up the smallest stable aggregate. Although heparin plays an important catalytic role in the nucleation reaction, its effects are included in the nucleation rate constant. The symbol $m(t)=m(0)-\sum_{j=n_{c}}^{\infty} j f(j, t)$ denotes the concentration of free monomer as a function of time, with $m(0)$ denoting the initial monomer concentration; $10 \mu \mathrm{M}$ for all experiments here. The concentration of fibrils containing $j$ monomeric residues is denoted $f(j, t)$, with $f(j, 0)=0$ for all $j \geq n_{\mathrm{c}}$ and $f(j, t)=0$ for all $j<n_{\mathrm{c}}$. Fibril elongation is described by the rate constant $k_{+}$, with dissociation of monomer from fibril ends governed by $k_{\text {off }}$ the factor of 2 allows for two free ends per fibril. Both elongation and dissociation rate constants are thus assumed independent of fibril length.

With careful treatment of boundary terms ${ }^{16,17}$, equation (3) can be summed to yield an expression for the zeroth moment of the fibril length distribution, $P(t)=\sum_{j=n_{\mathrm{c}}}^{\infty} f(j, t)$ :

$$
\dot{P}(t)=k_{\mathrm{n}} m(t)^{n_{\mathrm{c}}}
$$

We can formally integrate equation (4) to give

$$
P(t)=k_{\mathrm{n}} \int_{0}^{t} m\left(t^{\prime}\right)^{n_{\mathrm{c}}} \mathrm{d} t^{\prime},
$$

representing the total concentration of aggregates as a function of time.

Supplementary Fig. 8b-d illustrates how equation (5) can be used to obtain an upper bound for the average aggregate length distribution within this framework that is consistent with the kinetic available data for each K18 tau construct. For each data set, the time series for $m(t)$ can be inferred experimentally from measurements of the total concentration of soluble tau species, accounting for the known proportion of which is accounted for by oligomers (see Figs 3 and 4). The resulting numerical function is linearly interpolated (Supplementary Fig. 8b) and integrated to find $P(t)$ as per equation (5), with $k_{\mathrm{n}}$ set to the smallest possible value that ensures $P(t)$ accounts for observed oligomer populations (Supplementary Fig. 8c). This lower bound for $P(t)$ is then used to obtain an upper bound for the average aggregate length $\mu(t)$ as a function of time (Supplementary Fig. 8d), via the relation

$$
\mu(t)=(m(0)-m(t)) / P(t),
$$

where $m(0)-m(t)$ gives the total concentration of monomeric residues contained within aggregates.

By applying this procedure for a range of plausible critical nucleus sizes $\left(2 \leq n_{\mathrm{c}} \leq 10\right)$, we show that the maximum plausible average aggregate length allowed by classical nucleation-polymerization theory falls short of observed tau fibril lengths formed under these conditions ${ }^{23}$. In addition, the $n_{\mathrm{c}}=10$ case more generally demonstrates the failure of any model where monomers only form a single type of aggregate, provided that the total aggregate concentration does not decline beyond the time at which the observed oligomer population reaches a peak. Furthermore, by assuming a reasonable average fibril length of 2,000 monomer units throughout the reaction (a $1-\mu \mathrm{m}$ fibril with one tau molecule in each $\beta$-strand), the aggregate concentration $P(t)$ can be found numerically from equation (6), using the experimental time series for $m(t)$. These $P(t)$ curves thus represent strong upper bounds for the oligomer concentration $x(t)$, as shown overlaid in dotted black in Fig. 4b. Although at early reaction times this upper bound is underestimated due to transient smaller initial average aggregate size, at later times it is clear that classical nucleation-polymerization does not provide an adequate description. A modification to this basic theory is thus needed to reconcile observed oligomer concentrations with the production of long fibrils.

Conversion model and fitting. Oligomeric aggregates are now treated as distinct from fibrils, introducing an additional step in the reaction pathway: oligomers now exchange both with free monomer via a reaction of order $n_{\mathrm{c}}$ in the monomer concentration, and with fibrils via a reaction of first order in monomer concentration. Supplementary Fig. 10 and Fig. 4a illustrate this new model, demonstrating how it is coarse-grained, simplified and re-expressed in a useable form in terms of conversions between different species with established average sizes. The kinetic equations for this simple model read:

$$
\begin{gathered}
\dot{x}(t)=k_{\mathrm{n}} m(t)^{n_{c}}-\tilde{k}_{\mathrm{n}} x(t)-k_{+} m(t) x(t)+k_{\text {off }} f(t), \\
\dot{f}(t)=k_{+} m(t) x(t)-k_{\text {off }} f(t), \\
\dot{m}(t)=-x_{\mathrm{a}} k_{\mathrm{n}} m(t)^{n_{\mathrm{c}}}-\left(f_{\mathrm{a}}(t)-x_{\mathrm{a}}(t)\right) k_{+} m(t) x(t) \\
+x_{\mathrm{a}}(t) \tilde{k}_{\mathrm{n}} x(t)+\left(f_{\mathrm{a}}(t)-x_{\mathrm{a}}(t)\right) k_{\text {off }} f(t) .
\end{gathered}
$$

Concentrations of different populations are denoted with the symbols

$x(t)$ : oligomeric species,

$f(t)$ : fibrils,

$m(t)$ : monomers,

where the initial values $x(0)$ and $f(0)$ are 0 , and $m(0)=10 \mu \mathrm{M}$ as before. Heparin dependence is again subsumed into the nucleation rate constant. The critical nucleus size $n_{\mathrm{c}}$ is now taken as $n_{\mathrm{c}}=2$ for simplicity.

The various average lengths of different species, expressed as numbers of monomeric residues, are given by:

$x_{a}(t)$ : average length of oligomers,

$f_{a}(t)$ : average length of fibrils

and the various rate constants describe the processes shown in Fig. 4a and Supplementary Fig. 10b:

$k_{\mathrm{n}}$ : heparin-dependent nucleation of monomers to form oligomers,

$\tilde{k}_{\mathrm{n}}$ : complete dissociation of oligomers into monomers,

$k_{+}$: monomer-dependent conversion of oligomers to fibrils,

$k_{\text {off: }}$ conversion of fibrils back to small oligomers, releasing free monomer.

To conserve mass, the average lengths $x_{a}(t)$ and $f_{a}(t)$ must be constant with time. The oligomer length, averaged over the full reaction time, can be determined directly from the available data giving numbers of oligomers detected of each length. Average fibril length is estimated at 2,000 monomeric residues per fibril, although the quality of fit is not strongly sensitive to the precise value chosen.

The functions $m(t)$ and $x(t)$ are fitted directly to populations inferred from the data presented in Figs 3 and 4 , via numerical integration of the kinetic equations. All fits are numerical, with four rate constants fitted separately to each tau data set using a global least-squares Levenberg-Marquardt algorithm. The resulting best fits are shown in Fig. $4 \mathrm{~b}$

Seeding predictions. The effects of seeding are taken into account straightforwardly, by setting the initial population concentrations in equations (7-9) at $t=0$ according to the nature of the seeds. All rate constants are set according to the fitting procedure discussed above. For the simulations described in the main text, we investigate seeding by oligomers; $x(0)$ is set to the initial seed concentration, while $f(0)$ remains negligible. The rate of aggregation is monitored via the formation of fibril mass; if the unseeded reaction gives a final fibril residue concentration $f_{a} \cdot f(\infty)=W$, and at time $t_{W / 2}$ has produced a fibril residue 
concentration of $W / 2$, any increase in reaction rate on seeding is quantified by considering the time ${ }^{s} t_{W / 2}$ at which the seeded reaction produces a fibril residue concentration of $W / 2$. The seeding required to double the reaction rate is thus found by setting ${ }^{s} t_{W / 2} / t_{W / 2}=1 / 2$. For the K18 wild-type construct, this criterion is very similar to simply halving the reaction half-time. For the $\Delta \mathrm{K} 280$ deletion mutant, seeding leads to a non-negligible increase in the total fibril mass produced, thus motivating this more careful definition. Intracellular conditions are simulated by setting $m(0)=2 \mu \mathrm{M}$

Inferring activation free energies. For a given rate constant $k$ describing a particular reaction step, the activation free energy $\Delta G^{\ddagger}$ of the process at a temperature $T$ can be inferred from transition state theory ${ }^{44}$, via the equation

$$
k=\Gamma e^{-\Delta G^{\ddagger} /(R T)},
$$

where $R$ represents the molar gas constant. The prefactor $\Gamma$ has units matching those of $k$, and is approximately constant for a given process; it has a weak temperature dependence that to a good approximation is assumed negligible in the studied range. The change in activation energy $\Delta \Delta G^{\ddagger}$ for a given process on mutation can thus be found ${ }^{44}$ independently of $\Gamma$ by applying equation (10) to both the WT $\left(k_{\mathrm{WT}}\right)$ and the Mutant $\left(k_{\mathrm{Mut}}\right)$ system and subtracting:

$$
\Delta \Delta G^{\ddagger}=R T \ln \left(\frac{k_{\mathrm{WT}}}{k_{\mathrm{Mut}}}\right) .
$$

The values quoted in Table 1 are thus found by application of equation (11) to the four different rate constants in the conversion model.

\section{References}

1. Irwin, D. J., Lee, V. M. Y. \& Trojanowski, J. Q. Parkinson's disease dementia: convergence of alpha-synuclein, tau and amyloid-beta pathologies. Nat. Rev. Neurosci. 14, 626-636 (2013).

2. Lee, V. M. Y., Goedert, M. \& Trojanowski, J. Q. Neurodegenerative tauopathies. Annu. Rev. Neurosci. 24, 1121-1159 (2001).

3. Barghorn, S., Davies, P. \& Mandelkow, E. Tau paired helical filaments from Alzheimer's disease brain and assembled in vitro are based on beta-structure in the core domain. Biochemistry 43, 1694-1703 (2004).

4. Dehmelt, L. \& Halpain, S. The MAP2/Tau family of microtubule-associated proteins. Genome Biol. 6, 204 (2005).

5. Avila, J., Lucas, J. J., Perez, M. \& Hernandez, F. Role of tau protein in both physiological and pathological conditions. Physiol. Rev. 84, 361-384 (2004).

6. Jeganathan, S., von Bergen, M., Mandelkow, E. M. \& Mandelkow, E. The natively unfolded character of Tau and its aggregation to Alzheimer-like paired helical filaments. Biochemistry 47, 10526-10539 (2008).

7. Santarella, R. A. et al. Surface-decoration of microtubules by human Tau. J. Mol. Biol. 339, 539-553 (2004).

8. von Bergen, M., Barghorn, S., Biernat, J., Mandelkow, E. M. \& Mandelkow, E. Tau aggregation is driven by a transition from random coil to beta sheet structure. Biochim. Biophys. Acta 1739, 158-166 (2005).

9. Daebel, V. et al. beta-sheet core of tau paired helical filaments revealed by solidstate NMR. J. Am. Chem. Soc. 134, 13982-13989 (2012).

10. Barghorn, S. \& Mandelkow, E. Toward a unified scheme for the aggregation of tau into Alzheimer paired helical filaments. Biochemistry 41, 14885-14896 (2002).

11. Gustke, N., Trinczek, B., Biernat, J., Mandelkow, E. M. \& Mandelkow, E. Domains of Tau-protein and interactions with microtubules. Biochemistry 33, 9511-9522 (1994).

12. Goedert, M. et al. Assembly of microtubule-associated protein tau into Alzheimer-like filaments induced by sulphated glycosaminoglycans. Nature 383, 550-553 (1996).

13. Kampers, T., Friedhoff, P., Biernat, J. \& Mandelkow, E. M. RNA stimulates aggregation of microtubule-associated protein tau into Alzheimer-like paired helical filaments. FEBS Lett. 399, 344-349 (1996).

14. Ramachandran, G. \& Udgaonkar, J. B. Understanding the kinetic roles of the inducer heparin and of rod-like protofibrils during amyloid fibril formation by tau protein. J. Biol. Chem. 286, 38948-38959 (2011).

15. Bemporad, F. \& Chiti, F. Protein misfolded oligomers: experimental approaches, mechanism of formation, and structure-toxicity relationships. Chem. Biol. 19, 315-327 (2012).

16. Knowles, T. P. J. et al. An analytical solution to the kinetics of breakable filament assembly. Science 326, 1533-1537 (2009).

17. Cohen, S. I. A. et al. Nucleated polymerization with secondary pathways. I. Time evolution of the principal moments. J. Chem. Phys. 135, 065105 (2011).

18. Cremades, N. et al. Direct observation of the interconversion of normal and toxic forms of alpha-synuclein. Cell 149, 1048-1059 (2012).

19. Rizzu, P. et al. High prevalence of mutations in the microtubule-associated protein tau in a population study of frontotemporal dementia in the Netherlands. Am. J. Hum. Genet. 64, 414-421 (1999).
20. Barghorn, S. et al. Structure, microtubule interactions, and paired helical filament aggregation by tau mutants of frontotemporal dementias. Biochemistry 39, 11714-11721 (2000).

21. Lewis, J. et al. Neurofibrillary tangles, amyotrophy and progressive motor disturbance in mice expressing mutant (P301L) tau protein. Nat. Genet. 25, 402-405 (2000).

22. Horrocks, M. H. et al. Single Molecule Fluorescence under Conditions of Fast Flow. Anal. Chem. 84, 179-185 (2012).

23. von Bergen, M. et al. Mutations of tau protein in frontotemporal dementia promote aggregation of paired helical filaments by enhancing local betastructure. J. Biol. Chem. 276, 48165-48174 (2001).

24. Lasagna-Reeves, C. A., Castillo-Carranza, D. L., Guerrero-Munoz, M. J., Jackson, G. R. \& Kayed, R. Preparation and characterization of neurotoxic tau oligomers. Biochemistry 49, 10039-10041 (2010).

25. Lasagna-Reeves, C. A. et al. Tau oligomers impair memory and induce synaptic and mitochondrial dysfunction in wild-type mice. Mol. Neurodegener. 6, 39 (2011).

26. Lasagna-Reeves, C. A. et al. Alzheimer brain-derived tau oligomers propagate pathology from endogenous tau. Sci. Rep. 2, 700 (2012).

27. Oosawa, F. \& Asakura, S. Thermodynamics Of The Polymerization Of Protein (Academic Press, 1975).

28. Oosawa, F. \& Kasai, M. Theory of linear and helical aggregations of macromolecules. J. Mol. Biol. 4, 10-21 (1962).

29. Kumar, S. et al. Stages and conformations of the Tau repeat domain during aggregation and its effect on neuronal toxicity. J. Biol. Chem. 289, 20318-20332 (2014).

30. Eckermann, K. et al. The beta-propensity of Tau determines aggregation and synaptic loss in inducible mouse models of tauopathy. J. Biol. Chem. 282, 31755-31765 (2007).

31. Mocanu, M. M. et al. The potential for beta-structure in the repeat domain of tau protein determines aggregation, synaptic decay, neuronal loss, and coassembly with endogenous Tau in inducible mouse models of tauopathy. J. Neurosci. 28, 737-748 (2008).

32. Aoyagi, H., Hasegawa, M. \& Tamaoka, A. Fibrillogenic nuclei composed of P301L mutant tau induce elongation of P301L tau but not wild-type tau. J. Biol. Chem. 282, 20309-20318 (2007).

33. Chang, E. \& Kuret, J. Detection and quantification of tau aggregation using a membrane filter assay. Anal. Biochem. 373, 330-336 (2008).

34. Combs, B. \& Gamblin, T. C. FTDP-17 tau mutations induce distinct effects on aggregation and microtubule interactions. Biochemistry 51, 8597-8607 (2012).

35. Dinkel, P. D., Siddiqua, A., Huynh, H., Shah, M. \& Margittai, M. Variations in filament conformation dictate seeding barrier between three- and four-repeat tau. Biochemistry 50, 4330-4336 (2011).

36. Frost, B., Ollesch, J., Wille, H. \& Diamond, M. I. Conformational diversity of wild-type tau fibrils specified by templated conformation change. J. Biol. Chem. 284, 3546-3551 (2009).

37. Mohamed, N. V., Herrou, T., Plouffe, V., Piperno, N. \& Leclerc, N. Spreading of tau pathology in Alzheimer's disease by cell-to-cell transmission. Eur. J. Neurosci. 37, 1939-1948 (2013).

38. Frost, B. \& Diamond, M. I. Prion-like mechanisms in neurodegenerative diseases. Nat. Rev. Neurosci. 11, 155-159 (2010).

39. Orte, A., Clarke, R., Balasubramanian, S. \& Klenerman, D. Determination of the fraction and stoichiometry of femtomolar levels of biomolecular complexes in an excess of monomer using single-molecule, two-color coincidence detection. Anal. Chem. 78, 7707-7715 (2006).

40. Narayan, P. et al. Single molecule characterization of the interactions between amyloid-beta peptides and the membranes of hippocampal cells. J. Am. Chem. Soc. 135, 1491-1498 (2013).

41. Cohen, S. I. A. et al. Proliferation of amyloid-beta 42 aggregates occurs through a secondary nucleation mechanism. Proc. Natl Acad. Sci. USA 110, 9758-9763 (2013).

42. Ferrone, F. A., Hofrichter, J. \& Eaton, W. A. Kinetics of sickle hemoglobin polymerization .2. A double nucleation mechanism. J. Mol Biol. 183, 611-631 (1985).

43. von Bergen, M. et al. Assembly of tau protein into Alzheimer paired helical filaments depends on a local sequence motif ((306)VQIVYK(311)) forming beta structure. Proc. Natl Acad. Sci. USA 97, 5129-5134 (2000).

44. Fersht, A. Structure And Mechanism In Protein Science: A Guide To Enzyme Catalysis And Protein Folding, edn. 3 (W. H. Freeman \& Co Ltd., New York, USA, 1999).

\section{Acknowledgements}

D.K. acknowledges funding from the Wellcome Trust (WT089703) and MRC. E.M. acknowledges funding from the Wellcome Trust (WT089703), DZNE and Max-PlanckSociety. M.K. acknowledges fellowships from the Danish research council and the Lundbeck Foundation. N.S. and M.H.H acknowledge funding from the Augustus 
Newman foundation. G.A.G is funded by the Schiff Foundation. T.P.J.K acknowledges funding from the ERC, Augustus Newman Foundation and the BBSRC.

\section{Author contributions}

S.L.S. designed and performed the single-molecule fluorescence experiments, bulk kinetic assays, size-exclusion chromatography, SDS-PAGE and cell toxicity studies. S.L.S. also analysed the data and wrote the paper. G.A.G. developed the kinetic model and wrote the paper. S.K. designed and performed the electron microscopy studies. N.S. designed and performed the cell toxicity studies. M.K. designed and performed the single-molecule fluorescence studies. M.H.H. designed the single-molecule fluorescence studies. D.K., T.P.J.K and E.M supervised the project and edited the manuscript.

\section{Additional information}

Supplementary Information accompanies this paper at http://www.nature.com/ naturecommunications
Competing financial interests: The authors declare no competing financial interests.

Reprints and permission information is available online at http://npg.nature.com/ reprintsandpermissions/

How to cite this article: Shammas, S.L. et al. A mechanistic model of tau amyloid aggregation based on direct observation of oligomers. Nat. Commun. 6:7025 doi: $10.1038 /$ ncomms8025 (2015)

\section{(c) (1)}

This work is licensed under a Creative Commons Attribution 4.0 International License. The images or other third party material in this article are included in the article's Creative Commons license, unless indicated otherwise in the credit line; if the material is not included under the Creative Commons license, users will need to obtain permission from the license holder to reproduce the material. To view a copy of this license, visit http://creativecommons.org/ licenses/by/4.0/ 